\title{
La interpretación prejudicial recaída en el Proceso 242-IP-2015 del Tribunal de Justicia de la Comunidad Andina ${ }^{1}$
}

\author{
Preliminary Ruling 242-IP-2015 of the Andean Court of Justice
}

\author{
Juan Manuel Indacochea Jauregui \\ jmindacochea@gmail.com \\ Colaborador de la Magistratura del Perú en el Tribunal de Justicia de la Comunidad Andina. Ex \\ Consultor interno de la Magistratura de Bolivia del Tribunal de Justicia de la Comunidad Andina. \\ Master en Derecho Internacional en la Universidad Paris-Ouest Nanterre La Défense, Master \\ en Derecho de la Propiedad Intelectual en el CEIPI de la Universidad de Estrasburgo.
}

Resumen: La interpretación prejudicial recaída en el Proceso 242-IP-2015, sobre la marca táctil consistente en la superficie de la botella "Old Parr", constituye el primer pronunciamiento de un tribunal o corte internacional referido a una marca táctil. El propósito del presente artículo es explicar la aplicación de los principales requisitos de registrabilidad a los nuevos tipos de marca, a la luz de los criterios desarrollados en el citado pronunciamiento; así como el desarrollo jurisprudencial previo relativo a la legitimidad activa de las autoridades administrativas para solicitar interpretaciones prejudiciales facultativas, primer tema o cuestión tratada en la precitada interpretación prejudicial, en virtud de haber sido solicitada por la Dirección de Signos Distintivos de la Superintendencia de Industria y Comercio de Colombia.

Palabras clave: Derecho de marcas, juez nacional, marca táctil, principio de autonomía, principio de no funcionalidad, representación gráfica.

\begin{abstract}
The Preliminary Ruling 242-IP-2015, on the tactile mark consisting in the surface of the bottle "Old Parr", is the first decision rendered by an international court about tactile marks. The purpose of this paper is to explain the application of the main registration requirements to new types of Trademarks, in the light of the criteria explained in the aforementioned Preliminary Ruling. It also aims to analyze the previous jurisprudential development, concerning the active legitimacy of administrative authorities to optionally request preliminary rulings. That is the first question -or subject- addressed in the above-mentioned Preliminary Ruling since the interpretation was requested by an Administrative Authority -Office- of Colombia.
\end{abstract}

Keywords: Trademark Law, National Judge, Tactile Mark, Principle of Autonomy, Principle of Non-Functionality, Graphic Representation.

1 Artículo enviado el 30.12.2017 y aceptado el 05.07.2018.

Número de página no utilizable para citas

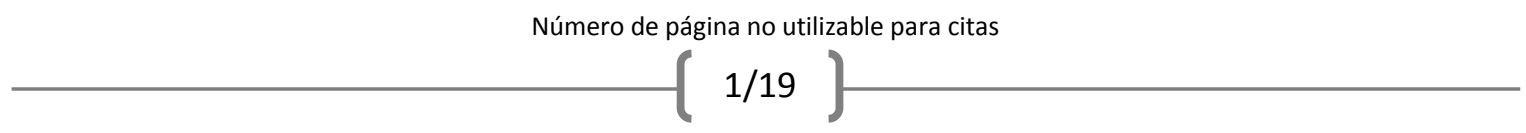


La interpretación prejudicial recaída en el Proceso 242-IP-2015 del Tribunal de Justicia de la Comunidad Andina Juan Manuel Indacochea Jauregui

\section{Introducción}

Los nuevos tipos de marcas usualmente se clasifican en signos visibles y signos no visibles ${ }^{2}$. En el caso particular de las marcas táctiles, estas pertenecen al grupo de los signos no visibles y es la superficie lo que da lugar a su reconocimiento y protección, por tratarse de una textura lo suficientemente arbitraria, original o particular como para identificar el origen empresarial de un producto determinado.

La jurisprudencia previa del Tribunal de Justicia de la Comunidad Andina (en adelante, el TJCA) no descartaba la registrabilidad de marcas constituidas por signos no visibles; únicamente establecía el requisito de perceptibilidad, entendido como "la necesidad de que un signo pueda ser apreciado por medio de los sentidos" 3 , así como el de representación gráfica, el cual será tratado in extenso más adelante.

Sin embargo, hasta el 24 de agosto de 2015, fecha del pronunciamiento objeto de estudio ${ }^{4}$, el único precedente administrativo de otorgamiento de una marca táctil, en un país miembro de la Comunidad Andina (en lo sucesivo, la CAN), lo comportaba la marca "Textura Old Parr" registrada a favor de Diageo Brands B.V. ${ }^{5}$, la misma solicitante que en el caso objeto del presente análisis, por parte del Instituto Ecuatoriano de la Propiedad Intelectual (en adelante, el IEPI). Sin embargo, en aquella oportunidad, se habría omitido solicitar al TJCA la correspondiente interpretación prejudicial ${ }^{6}$.

En relación con los signos no visibles, cabe destacar el registro de la marca de sonido consistente en el cornetín "D’Onofrio", registrada por el Instituto Nacional de Defensa de la Competencia y de la Protección de la Propiedad Intelectual (INDECOPI) de Perú a favor de D’Onofrio S.A.

2 Véase, al respecto, Juan Manuel Indacochea Jauregui, "Marcas no convencionales, no tradicionales, atípicas o simplemente nuevos tipos de marcas", Ius Inter Gentes, 11 (2015): 141-164, especialmente 146 y ss.

3 Interpretación prejudicial recaída en el Proceso 32-IP-97 de 2 de octubre de 1998, 10.

4 Interpretación prejudicial pronunciada en el Proceso 242-IP-2015 de 24 de agosto de 2015, marca: Textura superficie "Old Parr". A este respecto, cabe señalar que el autor colaboró, junto con la Dra. Angie Sasaki, en la elaboración del pronunciamiento objeto del presente estudio, la cual fue presentada -para su firma- por el Dr. Luis Diez Canseco como Magistrado ponente.

5 Solicitud 140058 de 17 de diciembre de 2003. Registro formalizado en virtud del Título 29597, de 28 de abril de 2004 , correspondiente a la marca táctil "Textura superficie Old Parr", para distinguir bebidas alcohólicas.

6 En cuanto a la interpretación prejudicial, es preciso recordar la sentencia bito dictada por el Tribunal de Justicia de las Comunidades Europeas (TJCE) en el caso Schwarze c/ Einfuhr- und Vorratsstelle für Getreide und Futtermittel, Asunto 16/65, de 1 de diciembre de 1965, en la cual el TJCE destacó que en virtud de la interpretación prejudicial el órgano jurisdiccional y el Tribunal de Justicia deben cooperar directa y recíprocamente, dentro del ámbito de sus propias competencias, a la elaboración de una resolución con el fin de garantizar la aplicación uniforme del derecho comunitario en todos los Estados miembros. Es justamente en virtud a la importancia de la interpretación prejudicial como mecanismo de cooperación fundamental para asegurar la aplicación uniforme del derecho comunitario, que la Doctrina sostiene que la misma comporta la "piedra angular" del sistema jurídico comunitario. Véase, en ese sentido, Ricardo Alonso García, Sistema jurídico de la Unión Europea (Pamplona: Thomson Reuters, 2010). Véase además, Juan Carlos Dueñas Muñoz, "La interpretación prejudicial, ¿piedra angular de la integración andina?”, Anuario de Derecho Constitucional Latinoamericano, 17 2011): 29-58, http://www20.iadb.org/intal/catalogo/PE/2012/10015.pdf [consultada: 6.jul.2017].

Número de página no utilizable para citas 
en virtud de la Resolución 11869-97-INDECOPI/OSD de 11 de septiembre de 1997. Sin embargo, para el otorgamiento del referido registro se habría obviado igualmente solicitar la respectiva interpretación prejudicial. En consecuencia, el pronunciamiento objeto del presente artículo constituye la primera oportunidad en que el TJCA se pronunció acerca de la registrabilidad de signos no visibles.

A nivel internacional, si bien ya se contaba con el registro de algunas marcas táctiles, como la funda violeta de la botella de Crown Royal, comercializada desde 1964", consistente en "la textura de terciopelo que cubre la superficie de una botella de vino" (del vino "Khvanchkara") '; de "un dispensador de fragancia personal esférico semejante a una pelota de baloncesto" "; con textura de algodón" para distinguir productos de jabón ${ }^{11}$; ninguno de estos registros supuso el pronunciamiento de un tribunal internacional. Por lo tanto, la interpretación prejudicial estudiada comporta la primera oportunidad en que un tribunal o corte internacional se manifiesta acerca de una marca táctil o de textura.

Por otra parte, es preciso tratar el tema de la legitimidad activa de las autoridades administrativas para solicitar interpretaciones prejudiciales facultativas por cuanto fue la Dirección de Signos Distintivos de la Superintendencia de Industria y Comercio (en lo sucesivo, la SIC) quien solicitó la interpretación prejudicial en el presente caso, autoridad cuyas decisiones son susceptibles de recurso judicial, vía contencioso-administrativa.

Es preciso explicar que, en virtud de lo dispuesto en el artículo 33 del Tratado de Creación del TJCA $^{12}$, los "jueces nacionales" que conozcan de un proceso en el que se deba aplicar disposiciones del ordenamiento jurídico comunitario "podrán” (verbo que denota facultad y no obligación) solicitar al TJCA la interpretación prejudicial facultativa de estas disposiciones, siempre que el ordenamiento jurídico interno prevea algún recurso contra la decisión emitida; de lo contrario, vale decir, de no existir posibilidad alguna de recurrir la futura decisión -firme- que dicte un determinado "juez nacional”, éste necesariamente deberá peticionar la correspondiente interpretación prejudicial obligatoria ${ }^{13}$.

7 Resolución 11869-97-INDECOPI/OSD de 11 de septiembre de 1997, emitida por la Oficina de Signos Distintivos del INDECOPI (Expediente administrativo 9730319). En la solicitud de registro, como descripción de la marca, se aprecia: "La melodía conforme a la impresión del pentagrama y cassettes adjuntos", descripción acompañada del correspondiente pentagrama. Solicitud de marca de 17 de enero de 1997, presentada por D’Onofrio S.A., para distinguir "helados comestibles, confitería, chocolates, galletas, caramelos y demás" productos comprendidos en la clase 30 de la

"Clasificación Internacional de Productos y Servicios para el Registro de Marcas" instituida por el Arreglo de Niza.

8 Registro 3, 137, 914 (USPTO) otorgado el 5 de septiembre de 2006 a favor de Diageo North America. Es preciso resaltar que la distintividad adquirida luego de cuatro décadas de uso en el mercado jugó un papel decisivo en este caso.

9 Registro 3, 155, 702 (USPTO) otorgado el 17 de octubre de 2006 a favor de American Wholesale Wine \& Spirits, Inc.

10 Registro 3, 348, 363 (USPTO) otorgado en 2007 a favor de Touchdown Marketing.

11 Registro 2, 682, 410 (USPTO) otorgado a favor de Fresh Inc.

12 Tratado de Creación del Tribunal de Justicia de la Comunidad Andina, suscrito el 28 de mayo de 1979 por los Estados de Perú, Bolivia, Ecuador, Colombia y Venezuela. Entrada en vigor: 19 de mayo de 1983. Fue codificado por la Decisión 472 de la Comisión de la Comunidad Andina.

13 Véase, por ejemplo, Roberto Salazar Manrique, "Fundamentos Jurídicos del Derecho Comunitario Andino (Documento de Trabajo)", en Memoria del Seminario Internacional: Integración Económica y Derecho Comunitario. Modelos Europeos y Americanos (Quito: Artes Gráficas Señal, 1997), 111.

Número de página no utilizable para citas 
La interpretación prejudicial recaída en el Proceso 242-IP-2015 del Tribunal de Justicia de la Comunidad Andina Juan Manuel Indacochea Jauregui

En consecuencia, la primera parte del presente artículo se encuentra consagrada al estudio de la evolución jurisprudencial del alcance del referido concepto autónomo -del derecho comunitario andino- de "juez nacional", es decir, de cómo y por qué el TJCA incorporó a dicho concepto otras entidades o autoridades distintas a los "jueces" en sentido estricto. Si bien el pronunciamiento objeto de estudio no constituye una jurisprudencia que aporta novedad alguna al respecto, sí es una de las primeras en reiterar los criterios para verificar tal legitimación, establecidos en la interpretación prejudicial pronunciada en el Proceso 121-IP-2014 ${ }^{14}$, el cual constituye la culminación de un destacable desarrollo jurisprudencial del TJCA ${ }^{15}$.

En efecto, la interpretación prejudicial recaída en el Proceso 121-IP-2014, comporta el ápice de la vasta jurisprudencia del TJCA en torno al concepto de "juez nacional”, contemplado en el ordenamiento jurídico comunitario andino, alcanzando a autoridades administrativas y estableciendo los criterios para verificar la legitimidad activa para solicitar interpretación prejudicial facultativa.

A efectos de un mejor análisis y entendimiento de la interpretación prejudicial recaída en el Proceso 242-IP-2015, objeto del presente ensayo, éste será dividido según las dos principales áreas del derecho tratadas en el referido pronunciamiento, la primera referida a la legitimidad activa de las autoridades administrativas para solicitar interpretación prejudicial facultativa; y, la segunda, a los requisitos de registrabilidad de la marca táctil o de textura. En la primera parte, se examinarán los lineamientos jurisprudenciales que se siguieron para poder finalmente admitir que autoridades administrativas soliciten interpretaciones prejudiciales facultativas. Mientras que, en la segunda parte, se analizarán los tres requisitos que suponen las mayores dificultades para el registro de marcas táctiles o de textura: El requisito de representación gráfica y la forma de publicación; la textura de uso común; y, la textura impuesta por una función técnica o por la naturaleza del producto.

\section{La legitimidad activa de las autoridades administrativas para solicitar interpretación prejudicial facultativa}

El TJCA comienza examinando la legitimidad activa de la entidad administrativa solicitante de la interpretación prejudicial, para lo cual, sigue los criterios expuestos en un pronunciamiento que comporta el ápice en lo que respecta a la legitimidad activa para solicitar interpretaciones

14 Proceso 121-IP-2014 de 20 de noviembre de 2014, originado en la consulta realizada el 22 de agosto de 2014 por la Dirección de Signos Distintivos del Instituto Nacional de Defensa de la Competencia y de la Protección de la Propiedad Intelectual (INDECOPI); Procedimiento administrativo interno: 541955-2013.

15 El primer pronunciamiento en reiterar los criterios establecidos en el Proceso 121-IP-2014, fue la interpretación prejudicial recaída en el Proceso 105-IP-2014 de 28 de noviembre de 2014, solicitada por el Servicio Nacional de Propiedad Intelectual (SENAPI) de Bolivia; procedimiento administrativo interno: 160313. 
prejudicial ante el TJCA: la interpretación prejudicial recaída en el precitado Proceso 121-IP2014 de 24 de agosto de 2015.

Es preciso admitir que, con anterioridad a la interpretación prejudicial emitida en el Proceso 121IP-2014, el TJCA ya venía interpretando el concepto de "juez nacional”, expresamente contenido en el artículo 33 del Tratado de Creación del TJCA - derecho primario de la CAN- y en los artículos 122 y 123 de su Estatuto ${ }^{16}$, de manera extensiva, comprendiendo cada vez más órganos decisorios dentro del referido concepto.

Sin embargo, fue precisamente con oportunidad de la interpretación prejudicial emitida en el Proceso 121-IP-2014, que no solo se incluyó a las autoridades administrativas, como la Dirección de Signos Distintivos de la SIC, autoridad solicitante de la interpretación prejudicial recaída en el Proceso 242-IP-2015 objeto de análisis, dentro del concepto de "juez nacional”, sino que además se establecieron los criterios para verificar su legitimación activa.

Según el TJCA, el concepto de “juez nacional” constituye un concepto autónomo ${ }^{17}$, propio del ordenamiento comunitario andino, susceptible de ser definido exclusivamente por el TJCA de acuerdo con criterios propios y tomando en cuenta el objeto ${ }^{18}$ de la interpretación prejudicial. En relación con la autonomía del ordenamiento jurídico comunitario cabe explicar que los principios -o características ${ }^{19}$ - que rigen el derecho comunitario son los siguientes: el principio de la "primacía"20; (ii) el principio de "eficacia directa"21; (iii) el principio de "aplicación inmediata",22; y, (iv) el principio de "autonomía"23; principios o características a los que el TJCA hace referencia en el párrafo 24 del pronunciamiento recaído en el Proceso 121-IP-2014.

Por su parte, Luis Ignacio Sánchez Rodríguez, al examinar la naturaleza del derecho primario y el derivado en la antigua Comunidad Económica Europea (CEE), concluye lo siguiente: "El derecho comunitario, en primer término, es autónomo a un mismo tiempo del derecho

16 Estatuto del Tribunal de Justicia del Acuerdo de Cartagena, aprobado mediante Decisión 184 el 19 de agosto de 1983 por la Comisión del Acuerdo de Cartagena.

17 En la interpretación prejudicial recaída en el Proceso 121-IP-2014, el TJCA comienza el análisis del primer acápite, referido a la legitimidad activa de la Dirección de Signos Distintivos y de la Comisión de Signos Distintivos del INDECOPI para solicitar interpretación prejudicial facultativa, haciendo referencia al carácter autónomo del derecho comunitario andino (párrafo 17).

18 En relación al objeto y finalidad de la institución de la interpretación prejudicial, es preciso remitirse a los artículos 32 del Tratado de Creación del TJCA y 121 de su Estatuto.

19 Fabián Novak Talavera, "La Comunidad Andina y su Ordenamiento Jurídico", en Derecho Comunitario Andino, por Allan R. Brewer Carías et al. (Lima: IDEI - Fondo Editorial PUCP, 2003), 57-100, especialmente 68 y ss.

20 También denominado principio de supremacía, de preeminencia, de prevalencia o de aplicación preferente. Véase, al respecto, la interpretación prejudicial, en la cual se sintetiza el extenso desarrollo jurisprudencial del TJCA en torno al principio de primacía del derecho comunitario, recaída en el Proceso 546-IP-2015, peticionada por el Tribunal Supremo de Justicia del Órgano Judicial de Bolivia, correspondiente al Expediente interno 1065-2014.

21 En relación a este principio (eficacia o efecto directo) del derecho comunitario andino, resulta oportuno remitir a la sentencia dictada en el Proceso 3-AI-96 de 24 de marzo de 1997, 14.

22 En relación a los principios de eficacia directa y aplicación inmediata, cabe precisar que, si bien este último principio o característica del derecho comunitario constituye la regla, ello no obsta para que se prevea lo contrario, como establece claramente el primer párrafo del artículo 3 del Tratado de Creación del TJCA.

23 Véase, por ejemplo, las decisiones pronunciadas en los Procesos 23-IP-2012 de 23 de agosto de 2012, 6; y 30-IP-2014 de 10 de septiembre de 2014, 8. Véase también, respecto al principio de autonomía en relación a procesos especiales, las sentencias dictadas en los Procesos 34-AI-01 de 21 de agosto de 2002, 49-50, y 1-AI-2001 de 27 de junio de 2002, 38. 
La interpretación prejudicial recaída en el Proceso 242-IP-2015 del Tribunal de Justicia de la Comunidad Andina Juan Manuel Indacochea Jauregui

internacional general y del derecho interno de los Estados Miembros"24. En tal virtud, el TJCA tiene la potestad de determinar el alcance de las disposiciones que conforman el ordenamiento jurídico comunitario andino y, en consecuencia, de principios y conceptos contenidos en tales disposiciones, llegando incluso -como veremos más adelante- más allá de lo establecido por el Tribunal de Justicia de la Unión Europea (TJUE).

En el pronunciamiento emitido en el Proceso 121-IP-2014, ante la consulta realizada por la Dirección de Signos Distintivos del INDECOPI, el TJCA estableció que el concepto de "juez nacional" constituye un "concepto autónomo del derecho comunitario" y, que por ello tenía la potestad de interpretarlo como un término genérico y comprensivo de todas las autoridades que administran justicia por mandato legal y que, al hacerlo deban aplicar o interpretar el ordenamiento comunitario andino.

Lo decidido es conforme a lo anteriormente manifestado por el propio TJCA en la interpretación prejudicial pronunciada en los Procesos 14-IP-2007 y 130-IP-2007²5, citada por el TJCA en el Proceso 121-IP-2014, que tuvieron origen en consultas planteadas por el Grupo de Trabajo de Competencia Desleal de la SIC. En aquella oportunidad, el TJCA recogió - una vez más- el criterio funcional, material u objetivo y no el criterio orgánico, formal o subjetivo, para concluir que necesariamente se debe ampliar el concepto de "juez nacional".

En efecto, el TJCA advierte que los países miembros pueden atribuir funciones jurisdiccionales a órganos diferentes del Poder Judicial para revestirlos de la competencia de aplicar normas jurídicas con la finalidad de resolver controversias y emitir decisiones firmes, es decir, el TJCA reconoce la creciente "jurisdiccionalización” de los procedimientos administrativos, como evidencia la misma naturaleza de los actos emitidos por órganos administrativos, para concluir afirmando la necesidad de incluirlos en el concepto de "juez nacional".

En tal virtud, el TJCA estableció en la interpretación prejudicial pronunciada en los Procesos 14-IP-2007 y 130-IP-2007 que en tanto se pretende la aplicación uniforme de la norma comunitaria (la finalidad de la interpretación prejudicial es garantizar la aplicación uniforme del ordenamiento jurídico comunitario), el concepto de "juez nacional” no debe circunscribirse sólo a los actos que emanan de los "jueces" en sentido estricto, sino también a los actos de otras autoridades que la aplican en los hechos.

La posibilidad de que los países miembros definieran libremente qué órgano nacional podía solicitar una interpretación prejudicial no solamente hubiera restringido el acceso al TJCA, sino que hubiera puesto en riesgo la aplicación uniforme del ordenamiento jurídico comunitario. Denegar a las autoridades administrativas poder efectuar la consulta facultativa ante el TJCA,

24 Luis Ignacio Sánchez Rodríguez, "Los Tratados Constitutivos y el Derecho Derivado", en Tratado de Derecho Comunitario Europeo, Tomo I, (Madrid: Ed. Civitas, 1986); citado, entre otras decisiones, en la recaída en el Proceso 1-IP-96, 9, de 9 de diciembre de 1996. El autor es citado, entre otras, en la interpretación prejudicial pronunciada en el precitado Proceso 546IP-2015, 7.

25 Procesos 14-IP-2007 de 21 de marzo de 2007 y 130-IP-2007 de 17 de octubre de 2007.

Número de página no utilizable para citas 
hubiera significado aceptar la aplicación de las normas andinas por parte de una autoridad que tuviera incertidumbre acerca de su alcance y, por consiguiente, el TJCA hubiera incumplido una de sus misiones esenciales, reconocida en el artículo 4 de su Estatuto, cual es aquella de asegurar la aplicación e interpretación uniforme del derecho comunitario en todos los países miembros.

A este respecto, cabe recordar que la interpretación prejudicial constituye un mecanismo de cooperación entre los "jueces nacionales" y el TJCA, que tiene como finalidad asegurar la aplicación uniforme del derecho comunitario andino ${ }^{26}$. Asimismo, es preciso tener en cuenta que, de permitirse que los tribunales nacionales interpreten de manera unilateral el derecho comunitario conforme a su derecho interno, evidentemente se podrían generar -en la prácticados o más interpretaciones divergentes, hechos que atentarían contra la uniformidad que debe primar en todo ordenamiento jurídico ${ }^{27}$.

Adicionalmente, se debe reconocer que no todas las resoluciones administrativas son impugnadas en la vía judicial, y por tanto, existía el riesgo de que se consolidaran actos que contravinieran la jurisprudencia del TJCA y fuesen contrarios al ordenamiento jurídico comunitario. En la práctica, el incumplimiento de la obligación de solicitar interpretación prejudicial obligatoria generó a su vez el incumplimiento por parte de un país miembro, por no anular laudos arbitrales expedidos sin mediar la correspondiente solicitud de interpretación prejudicial obligatoria ${ }^{28}$; toda vez que los Estados son los sujetos principales del derecho comunitario y son precisamente ellos quienes responden por los actos de sus órganos o instituciones.

Cabe comentar que, el TJCA ya había hecho referencia a los Procesos 14-IP-2007 y 130-IP-2007, al desarrollar la cuestión relativa al "alcance del término juez nacional" en una decisión a propósito de una acción de incumplimiento interpuesta por la Empresa de Telecomunicaciones de Bogotá S.A. (ETB S.A. E.S.P.) contra Colombia ${ }^{29}$. Dicha decisión es, a su vez, citada en otras decisiones, como por ejemplo, por la recaída en el Proceso 57-IP-2012 ${ }^{30}$, cuando el TJCA desarrolla la cuestión referida al "árbitro o tribunal de arbitramento como juez nacional”.

En la interpretación prejudicial recaída en el Proceso 57-IP-2012, se estableció que si los árbitros tienen funciones jurisdiccionales y actúan "en última instancia” - este criterio, en particular,

26 Respecto a la finalidad de asegurar la "aplicación uniforme" del derecho comunitario, es oportuno remitir al punto 3.3 de la interpretación prejudicial hito pronunciada por el TJCA en el Proceso 1-IP-87 de 3 de diciembre de 1987, 4-5. Véase además, Elizabeth Salmón Gárate, "Evolución institucional de la Comunidad Andina: perspectivas y problemas", en Derecho Comunitario Andino, Allan R. Brewer Carías et al. (Lima: IDEI - Fondo Editorial PUCP, 2003), 21-55, especialmente 44.

27 En ese sentido, Fabián Novak Talavera, "La Comunidad Andina y su Ordenamiento Jurídico", 96; quien cita asimismo a José Guillermo Andueza, "La Interpretación Prejudicial y el Tribunal de Justicia del Acuerdo de Cartagena”, en El Tribunal de Justicia del Acuerdo de Cartagena (Montevideo: ALADI, 1985 / Buenos Aires: INTAL, Banco Interamericano de Desarrollo, 1986), 104.

28 Sentencia dictada el 26 de agosto de 2011 en el Proceso 03-AI-2010.

29 Sentencia pronunciada el 26 de agosto de 2011 en el Proceso 3-AI-2010.

30 Interpretación prejudicial recaída en el Proceso 57-IP-2012 de 11 de julio de 2012. 
La interpretación prejudicial recaída en el Proceso 242-IP-2015 del Tribunal de Justicia de la Comunidad Andina Juan Manuel Indacochea Jauregui

habría sido aplicable únicamente para la interpretación prejudicial obligatoria ${ }^{31}-\mathrm{y}$ no dependen de los jueces nacionales en sentido estricto, para efectos de la norma comunitaria actúan como "jueces nacionales".

En dicha oportunidad, al igual que en los precitados Procesos 14-IP-2007 y 130-IP-2007, se realizó una interpretación extensiva del concepto de “juez nacional”, comprendiendo a los árbitros que aplican el derecho comunitario andino. Por consiguiente, se permitió que éstos soliciten la correspondiente interpretación prejudicial al TJCA de manera directa, sin que sea necesaria la mediación de organismos judiciales. De esta manera, surgió -vía interpretativa-la mencionada obligación de los árbitros o tribunales de arbitramento de solicitar interpretaciones prejudiciales obligatorias.

En suma, es posible afirmar que la jurisprudencia del TJCA relativa al alcance del concepto de "juez nacional", se erigió principalmente sobre la base de cuatro pilares fundamentales: (i) el principio de Autonomía del derecho comunitario andino, gracias al cual puede definir los límites del referido concepto; (ii) el objeto y finalidad de la interpretación prejudicial, cual es -según el artículo 32 del Tratado de Creación del TJCA y el artículo 121 de su Estatuto- asegurar la aplicación uniforme de dicho derecho en el territorio de los países miembros; (iii) la función del TJCA -explicitada en el artículo 4 de su Estatuto- de asegurar la aplicación e interpretación uniforme del derecho comunitario en todos los Estados miembros; y, (iv) la naturaleza de los actos emitidos por las autoridades administrativas, analizada siguiendo un criterio funcional, material u objetivo, habida cuenta asimismo de la creciente "jurisdiccionalización" de los procedimientos tramitados en sede administrativa.

Por otra parte, es importante destacar que el incumplimiento de la obligación impuesta por el ordenamiento jurídico comunitario ${ }^{32}$, de solicitar al TJCA la interpretación prejudicial de normas comunitarias por parte de jueces o árbitros, es una causal de anulación de laudos arbitrales, lo cual se evidencia en sentencias ${ }^{33}$ del Consejo de Estado de Colombia, por medio de las cuales se adoptaron decisiones definitivas en relación con el correspondiente fallo del TJCA ${ }^{34}$, que ordenaban "dejar sin efectos" las sentencias que resolvieron recursos extraordinarios de anulación contra laudos arbitrales que dirimieron las controversias surgidas entre Empresa de

31 Véase, al respecto, el primer párrafo de las Conclusiones de la interpretación prejudicial recaída en el Proceso 57-IP-2012 de 11 de julio de 2012 .

32 A este respecto, cabe citar la interpretación prejudicial recaída en el Proceso 2-IP-91 de 26 de febrero de 1991, 3, en la cual el TJCA manifestó que: "es obligación del juez nacional constatar si dentro del proceso a su cargo resulta previsible que deban aplicarse normas comunes a fin de decidir el proceso, antes de proceder a solicitar su interpretación prejudicial."

33 Véase, por ejemplo, las sentencias dictadas por la Sección Tercera de la Sala de lo Contencioso Administrativo del Consejo de Estado de Colombia el 9 de agosto de 2012 en los Expedientes -con Radicación- 11001032600020120001300 (43.045) y 11001032600020120001800 (43.195).

34 Véase, Dictamen 02-2010 de la Secretaría de la Comunidad Andina, de 24 de marzo de 2010. Véase además, la Sentencia del TJCA dictada en el Proceso de acción de incumplimiento 3-AI-2010, de 26 de agosto de 2011, en la cual determinó el incumplimiento de la República de Colombia.

Número de página no utilizable para citas 
Telecomunicaciones de Bogotá y operadores particulares ${ }^{35}$, o que incluso declararon la nulidad de los laudos arbitrales ${ }^{36}$.

Con anterioridad a el pronunciamiento emitido en el Proceso 121-IP-2014, el TJCA ya había producido un vasto desarrollo jurisprudencial a fuerza de admitir solicitudes de interpretaciones prejudiciales planteadas por diferentes autoridades, que no ocupaban el cargo de "jueces" en sentido estricto, tales como el Tribunal Administrativo del Atlántico ${ }^{37}$, el Grupo de Trabajo de Competencia Desleal de la $\mathrm{SIC}^{38}$, los Tribunales Arbitrales de la Cámara de Comercio de Bogotá $^{39}$, el Centro de Arbitraje de la Cámara de Comercio de Medellín ${ }^{40}$ y el Centro de Arbitraje de la Cámara de Comercio Ecuatoriana - Americana ${ }^{41}$.

Sin embargo, la interpretación prejudicial emitida en el Proceso 121-IP-2015 ${ }^{42}$ comportó la primera oportunidad en la cual el TJCA reconoció la legitimidad activa de una Oficina de Propiedad Industrial o Intelectual para solicitar interpretación prejudicial facultativa; reconociéndose de esta manera su rol principal en la promoción y garantía de los derechos reconocidos en los Regímenes Comunes de Propiedad Industrial ${ }^{43}$, sobre Derechos de Autor y Derechos Conexos ${ }^{44}$, de Protección de los Derechos de los Obtentores Vegetales ${ }^{45}$ y de Acceso a los Recursos Genéticos ${ }^{46}$, normativas comunitarias que expresamente indican que son precisamente las oficinas nacionales competentes las encargadas de su aplicación.

Cabe destacar que, en la primera cuestión prejudicial o tema desarrollado (mal llamado "tema objeto de interpretación" ${ }^{47}$ ) en el pronunciamiento en el precitado Proceso 121-IP-2014, a fin de verificar la legitimidad activa de la Dirección de Signos Distintivos y la Comisión de Signos Distintivos del INDECOPI para solicitar interpretación prejudicial facultativa, el TJCA no se limita a seguir la jurisprudencia en torno al concepto -autónomo del derecho comunitario- de “juez nacional”, sino que establece requisitos para tal verificación, siguiendo en gran medida a la

35 Véase, al respecto, Natalia Alarcón Pérez, Aspectos procedimentales de la interpretación prejudicial ante el Tribunal de Justicia de la Comunidad Andina (Medellín: Universidad CES, Facultad de Derecho), 16.

36 Véase, por ejemplo, la sentencia pronunciada por la Sección Tercera de la Sala de lo Contencioso Administrativo del Consejo de Estado el 2 de diciembre de 2015.

37 Interpretación prejudicial emitida en el Proceso 30-IP-98 de 16 de junio de 1999.

38 Decisiones pronunciadas en los Procesos 14-IP-2007 de 21 de marzo de 2007 y 130-IP-2007 de 17 de octubre de 2007.

39 Decisiones recaídas en los Procesos 161-IP-2013 de 13 de mayo de 2014, 181-IP-2013 de 13 de mayo de 2014 y 14-IP2014 de 13 de mayo de 2014.

40 Interpretación prejudicial expedida en el Proceso 79-IP-2014 de 25 de agosto de 2014.

41 Interpretación prejudicial pronunciada en el Proceso 262-IP-2013 de 20 de marzo de 2014.

42 Interpretación prejudicial que fue seguida y reiterada por la recaída en el precitado Proceso 105-IP-2014 de 28 de noviembre de 2014, solicitada por el SENAPI de Bolivia.

43 Decisión 486 de la Comisión de la Comunidad Andina, de 14 de septiembre de 2000.

44 Decisión 351 de la Comisión del Acuerdo de Cartagena, de 17 de diciembre de 1993.

45 Decisión 345 de la Comisión del Acuerdo de Cartagena, de 21 de octubre de 1993.

46 Decisión 391 de la Comisión del Acuerdo de Cartagena, de 2 de julio de 1996.

$47 \mathrm{Al}$ respecto, es importante aclarar que el TJCA interpreta en vía prejudicial (i.e., el objeto de una interpretación prejudicial) son "las normas que conforman el ordenamiento jurídico de la Comunidad Andina" (Artículo 32 del Tratado de Creación y Artículo 121 del Estatuto del TJCA).

Número de página no utilizable para citas 
La interpretación prejudicial recaída en el Proceso 242-IP-2015 del Tribunal de Justicia de la Comunidad Andina Juan Manuel Indacochea Jauregui

jurisprudencia Vaassen ${ }^{48}$ del Tribunal de Justicia de las Comunidades Europeas (TJCE), ahora TJUE.

En dicha oportunidad, el TJCE concluyó -luego de comprobar el cumplimiento de los citados criterios- respecto de la primera cuestión prejudicial, relativa a la admisibilidad de la petición de interpretación, que: "Considerando que, en estas circunstancias, procede considerar al Scheidsgerecht como órgano jurisdiccional en el sentido del Artículo 177; que, por tanto, procede admitir la petición de interpretación" ${ }^{\prime 4}$.

En consecuencia, el TJCA estableció criterios, para determinar la legitimidad activa, sobre una base analítica similar a la empleada en la denominada jurisprudencia $V$ aassen, desarrollada por el TJCE en la sentencia del caso Dorsch Consult ${ }^{0}$, decisiones en las cuales dicho Tribunal formuló los siguientes criterios para determinar si el término "órgano jurisdiccional", en el sentido del artículo 177 del Tratado constitutivo de la $\mathrm{CEE}^{51}$, puede incluir, en determinadas condiciones, un organismo que no sea un Tribunal ordinario: “[...] el origen legal del órgano, su permanencia, el carácter obligatorio de su jurisdicción, el carácter contradictorio del procedimiento, la aplicación por parte del órgano de normas jurídicas, así como su independencia." 52

De esta manera, el TJCA habría importado y adaptado los referidos criterios, a efecto de verificar la legitimidad activa de entidades o autoridades nacionales solicitantes, enunciándolos en el siguiente orden: (i) origen legal y permanencia del órgano consultante; (ii) carácter obligatorio de su jurisdicción; (iii) carácter contradictorio del procedimiento; (iv) aplicación por parte del órgano consultante de normas jurídicas comunitarias; (v) independencia.

48 TJCE, sentencia del Tribunal de Justicia de 30 de junio de 1966, Asunto 61/65, caso Viuda de G. Vaassen-Göbbels c/ Dirección del Beambtenfonds voor het Mijnbedrijf. Consulta formulada por el Scheidsgerecht van het Beambtenfonds voor het Mijnbedrijf de Heerlen (Holanda), Tribunal del Fondo de Pensiones de los Trabajadores Mineros de Heerlen, institución a la cual se reconoció legitimidad para formular la consulta,

49 TJCE, sentencia de 30 de junio de 1966.

50 TJCE, sentencia del Tribunal de Justicia de 17 de septiembre de 1997, Asunto C-54/95, caso Dorsch Consult, párrafo 23; consulta formulada por la Vergabeiberwachungsausschuß des Bundes (Comisión Federal de Control de la Adjudicación de Contratos Públicos), institución a la que se reconoció legitimidad para formular la consulta). Véase además, la sentencia de 21 de marzo de 2000, Asuntos acumulados C-110/98 a C-147/98, caso Gabalfrisa, en el cual el TJCE se pronunció a favor del carácter de "órgano jurisdiccional” del Tribunal Económico-Administrativo Regional de Cataluña

51 Tratados de Roma ("Tratado constitutivo de la Comunidad Económica Europea"), firmados en Roma el 25 de marzo de 1957. Entrada en vigor: 1 de enero de 1958.

52 Caben destacar dos casos adicionales en el ámbito comunitario europeo: (a) la Cour d'Arbitrage de Bélgica envió una consulta sobre la interpretación de determinadas disposiciones normativas de la Directiva 93/16/CEE del Consejo, de 5 de abril de 1993; y, (b) el Tribunal Constitucional de Hesse de Alemania remitió, en el marco de un control de constitucionalidad, una solicitud prejudicial sobre la Directiva 76/207/CEE, de 9 de febrero de 1976. Si bien en ambos casos el TJCE se pronunció acerca del alcance del concepto de "órgano jurisdiccional nacional", comprendiendo a los Tribunales Constitucionales nacionales, en ninguno de ellos reparó sobre la función jurisdiccional que ejercían los Tribunales Constitucionales solicitantes. Véase, sentencia del TJCE de 19 de febrero de 1997, caso Cour d'Arbitrage, Asunto 6/97, "Fédération Belge des Chambres Syndicales de Médecins s/annulation de l'article 4, \$ 2, du decret de la Communanté Flamande du 5 de avril de 1995", publicada en Recopilación de Jurisprudencia de la Cour d'Arbitrage 1 (enerofebrero, 1-8/97), 77. 


\section{Los requisitos de registrabilidad de la marca táctil o de textura}

La Decisión 486 establece ciertas causales de irregistrabilidad de marcas que comportan verdaderos límites al registro de nuevos tipos de marcas -y no solo al registro de marcas táctiles, tales como la prohibición de registro como marcas de signos que consistan exclusivamente en formas usuales de los productos o de sus envases, o en formas o características impuestas por la naturaleza o la función de dicho producto (artículo 135 literal c), así como aquellos que supongan únicamente formas u otros elementos que den una ventaja funcional o técnica al producto al cual se aplican (artículo 135 literal d). Asimismo, el requisito de representación gráfica, contemplado en el primer párrafo del artículo 134, plantea por su parte una seria dificultad para el registro de este tipo de marcas, al igual que la forma de la publicación de la solicitud.

En consecuencia, el desafío en relación a este tipo de marcas lo comporta no solo el requisito formal de la representación gráfica, sino también el denominado requisito de no funcionalidad y el relativo a la proscripción de signos (e.g., gráfico, forma, textura, sonido, olor, etc.) de uso común para distinguir determinados productos o servicios, como causales de irregistrabilidad de marcas por falta de distintividad intrínseca. Por lo tanto, a partir de la segunda cuestión analizada en la interpretación prejudicial emitida en el Proceso 242-IP-2015, objeto del presente análisis, fueron desarrollados particularmente estos requisitos.

En cuanto a "la forma de publicación de una marca táctil o de textura", es conveniente asociarla al requisito de susceptibilidad de representación gráfica. En primer lugar, por cuanto el propio TJCA hace referencia a este requisito cuando trata el mismo tema. En segundo lugar, debido a que, como lo deja inferir el TJCA, el requisito formal de representación gráfica resulta fundamental justamente en virtud de la necesidad de publicación de la marca solicitada, en aras de proteger derechos de terceros interesados, como competidores o consumidores.

\subsection{E1 requisito de representación gráfica y la forma de publicación}

El primer desafío que plantean los signos no visibles radica en el cumplimiento del requisito formal de "representatividad gráfica", prescrito en el primer párrafo del artículo 134 de la Decisión $486^{53}$. En relación con el procedimiento de registro, los signos no visibles plantean el problema práctico de su representación gráfica o de otro tipo, que constituye un requisito en muchas legislaciones de marcas. El requisito de susceptibilidad de representación gráfica, requerido por diversas normativas nacionales y supranacionales, en realidad no es obligatorio

53 Véase, respecto al criterio formal de representación gráfica, la interpretación prejudicial emitida en el Proceso 32-IP-97 de 2 de octubre de 1998, 10, que cita a Marco Matías Aleman, "Marcas" (Bogotá: Top Management), 77. 
La interpretación prejudicial recaída en el Proceso 242-IP-2015 del Tribunal de Justicia de la Comunidad Andina Juan Manuel Indacochea Jauregui

según el Acuerdo sobre los Aspectos de los derechos de Propiedad Intelectual relacionados con el Comercio (Acuerdo sobre los ADPIC) ${ }^{54}$.

En los párrafos 54 y 55 del pronunciamiento objeto de estudio, el TJCA hace referencia a las decisiones Shield Mark BV ${ }^{55}$, del TJCE, y Qualitex ${ }^{56}$, de la Corte Suprema de Estados Unidos de América. En la primera decisión, el TJCE estableció que la lista de signos contenida en el artículo 2 de la Directiva 2008/95/EC no era exhaustiva y que los signos no visibles no están expresamente excluidos de la Directiva. Por su parte, la sentencia de la Corte Suprema estadounidense dispuso que las disposiciones contenidas en el Lanham Act ${ }^{57}$ no pueden ser interpretadas de manera restrictiva y definió al signo ${ }^{58}$ como todo aquello capaz de transmitir un significado usado por seres humanos ${ }^{59}$.

El TJCA, en el párrafo 91 de la interpretación prejudicial comentada, realiza una referencia fundamental al principio de precisión, desarrollado previamente por la jurisprudencia comunitaria europea $^{60}$, explicando la necesidad del recurso a nuevas técnicas para hacerlo efectivo, y señalando contundentemente que la representación gráfica no es sólo un mero requisito técnico para el registro de marcas, sino que supone una manifestación del comentado principio, el cual permite la identificación precisa del signo que se solicita a registro y, en consecuencia, del objeto de una eventual protección por parte del derecho de marcas.

En el párrafo siguiente, el TJCA recoge los criterios jurisprudenciales europeos establecidos en el caso Sieckman ${ }^{61}$, es decir, que el referido principio de precisión supone que la representación gráfica del signo debe ser clara, precisa, completa en sí misma, inteligible -para otros consumidores y fabricantes-, duradera y objetiva. A este respecto, según afirma el TJCA en el párrafo 95 de la interpretación prejudicial comentada, el requisito de representación gráfica permite una definición precisa de la marca, permitiendo que, tanto las autoridades administrativas como las judiciales, el titular de la marca, los consumidores y los competidores puedan conocer el alcance de la protección otorgada o solicitada a registro.

Adicionalmente, el TJCA, siguiendo los criterios recogidos por la OMPI ${ }^{62}$, sostuvo - en el párrafo 99 de la interpretación prejudicial estudiada- que la representación gráfica del signo puede

\footnotetext{
54 Artículo 15 del Acuerdo sobre los Aspectos de los Derechos de Propiedad Intelectual relacionados con el Comercio (Acuerdo sobre los ADPIC), Anexo 1C del Acuerdo por el que se establece la Organización Mundial del Comercio (OMC) firmado en 1994.

55 Sentencia del TJCE 27 de noviembre de 2003, Asunto C-283/01, caso Shield Mark BV.

56 Caso Qualitex Co. c/ Jacobson Products Co., Inc., 514 US. 159, 34 USPQ.2d 1161 (1995).

57 El Lanbam Act, de 1946, es también conocido como Trademark. Act o Ley de marcas estadounidense.

58 "Symbol” o “device" según el Lanham Act $\$ 45$.

59 Caso Qualitex Co. c/ Jacobson Products Co., \$162.

60 Véase, entre otras, la sentencia del TJCE de 24 de junio de 2004, Asunto C-49/02, caso Heidelberger Bauchemie GmbH, E.T.M.R. 99, $\$ 13$.

61 Sentencia del TJCE de 12 de diciembre de 2002, Asunto C-273/00, caso Ralf Sieckmann c/ Deutsches Patent-und Markenamt (marca olfativa).

62 Véase en ese sentido, el documento preparado por la Secretaría del Comité Permanente sobre el Derecho de Marcas, Diseños Industriales e Indicaciones Geográficas de la OMPI, Nuevos tipos de marcas, Documento SCT/16/2, de 1 septiembre de 2006, 11.
} 
plasmarse mediante impresión en relieve (i.e., sistema braille). El TJCA considera que es preferible contar con múltiples representaciones de la marca, tomadas en perspectivas diferentes y según distintos métodos y modalidades.

Así, el TJCA explica -en el párrafo 98- que mientras más representaciones diferentes de una marca táctil o de textura sean publicadas, se comunicará con mayor precisión lo reivindicado y así se evitarán futuras controversias relativas a la marca táctil o de textura solicitada a registro. En efecto, en relación al procedimiento empleado para representar gráficamente al signo solicitado, los nuevos tipos de marcas ofrecen frecuentemente posibilidades diversas, que varían, por ejemplo, entre la descripción por escrito y la reproducción digital.

Adicionalmente, en el párrafo siguiente, el TJCA considera que la descripción simple permite al examinador determinar prima facie lo que se pretende registrar, por cuanto la inspección visual de la marca generalmente no sirve per se para determinar el objeto reivindicado con precisión o certitud en el caso de los signos no visibles. En efecto, el TJCA admite que la descripción simple también permite darse cuenta de ciertas características de la marca que podrían no aparecer en los gráficos que pretendan representar la marca. Ello resulta determinante particularmente en el caso de las marcas táctiles debido a que ciertos tipos de textura son casi imposibles de recrear mediante simples gráficos ${ }^{63}$.

En suma, en la interpretación prejudicial estudiada, el TJCA estableció que, en primer lugar, se deberá contar con la descripción clara, precisa y completa de la textura, incluyendo un dibujo tridimensional o fotografía. En segundo lugar, se deberá presentar una muestra física del objeto que contiene la textura, debiendo las oficinas nacionales competentes permitir el acceso a dichos objetos cuando sea solicitado. Finalmente, como se comentó anteriormente, el TJCA recomendó anexar una representación en código braille de la marca táctil que se solicita a registro.

\subsection{La textura de uso común}

La causal de irregistrabilidad contemplada en la primera proposición gramatical del enunciado del literal c) del artículo 135 supone la prohibición de registro del signo consistente exclusivamente en "formas" usuales de los productos o de sus envases, causal frecuentemente alegada en relación a solicitudes de marcas tridimensionales, debido a que, de otorgarse un derecho exclusivo sobre las formas usuales de los productos, se limitaría a los demás competidores la posibilidad de usar formas genéricas, con lo cual se bloquearía el acceso al respectivo mercado o sub-mercado de nuevos competidores ${ }^{64}$.

63 Cabe destacar que en los países del Benelux se han registrado algunas marcas gustativas como, por ejemplo, el "sabor de regaliz" para distinguir productos de papel e impresión, marca que fue representada mediante una descripción escrita. Asimismo, cabe citar la sentencia del TJCE de 11 de febrero de 1999, caso Vennootschap Onder Firma Senta Aromatic Marketing's Application (Holanda), Asunto R 156/1998-2, Fundamentos.

64 Véase, en ese sentido, la interpretación prejudicial pronunciada en el Proceso 84-IP-2003 de 5 de octubre de 2003; la cual es citada, entre otras, en la interpretación prejudicial emitida en el Proceso 195-IP-2007 de 5 de marzo de 2008, 15. 
La interpretación prejudicial recaída en el Proceso 242-IP-2015 del Tribunal de Justicia de la Comunidad Andina Juan Manuel Indacochea Jauregui

A este respecto, el TJCA realiza una acertado análisis de la causal de irregistrabilidad absoluta contenida en la primera proposición del literal (c) del artículo 135 de la Decisión 486, recurriendo a la analogía pero respetando el espíritu de la norma, la ratio legis, es decir, interpreta que "formas" usuales también puede referirse a "texturas" usuales o de uso común. En efecto, el TJCA realiza una interpretación extensiva de la causal que prohíbe el registro de un producto o su envase con características usuales, dando a entender que la causal que impide registrar la "forma" de uso común de un producto o su envase debe entenderse como todo aspecto externo del producto y no únicamente su envase-, susceptible de ser captado por los consumidores, incluida la "textura" del producto -así como de su envase o cualquier continente-, en otras palabras, el TJCA incluye a las "texturas" de uso común dentro del supuesto de la norma.

Siguiendo la línea interpretativa establecida en interpretaciones prejudiciales precedentes, el TJCA explica que las "formas" o "texturas" -intercambiando ambos conceptos mediante una interpretación sistemática y teleológica o finalista- de uso común son aquellas que, de manera frecuente y ordinaria se emplean en el mercado en relación con una clase internacional de productos determinada, de manera tal que el consumidor no relaciona tales "formas" o "texturas" con un origen empresarial específico, ya que en su mente estas se relacionan con el género de productos y no con un origen empresarial determinado.

Para concluir el presente acápite, conviene recordar que, como viene reiterando el TJCA en su jurisprudencia, las formas usuales o de uso común deben apreciarse en relación con los productos -o servicios- que ampare el signo cotejado. Es decir, lo que es usual o de uso común en una determinada clase internacional de productos o servicios puede no serlo en otra, salvo que exista similitud o conexión competitiva ${ }^{65}$ entre ambas clases de productos - o servicios ${ }^{66}$.

\subsection{La textura impuesta por una función técnica o por la naturaleza del producto}

En relación con los nuevos tipos de marcas, se contemplan los requisitos de no funcionalidad y que la forma reivindicada no sea de uso común para distinguir los productos o servicios que se pretende amparar. En efecto, en el artículo 135 de la Decisión 486, literales (c) y (d), se ha optado por contemplar, como causales de irregistrabilidad absoluta, los signos que consistan exclusivamente en "formas" usuales de los productos o de sus envases, en "formas" o características impuestas por la naturaleza o la función de dicho producto o del servicio de que

65 Véase, la interpretación prejudicial recaída en el Proceso 541-IP-2015, correspondiente al Expediente interno 2013-00028, en la cual se establecieron con precisión los criterios actuales para examinar la conexión competitiva.

66 De igual manera, la determinación de la debilidad o fortaleza del carácter distintivo -grado de distintividad- del signo dependerá de la capacidad de la textura contenida en el mismo de identificar el origen empresarial del producto o servicio correspondiente, apreciada desde la perspectiva del consumidor pertinente; lo cual puede resultar determinante a efectos de verificar la comisión de una infracción al derecho de marcas. Por ejemplo, es posible mencionar el caso del bolígrafo "Waterman", en el cual el Tribunal Regional Superior de Frankfurt (Alemania) consideró que el clip del bolígrafo, que mostraba una típica división longitudinal, era percibido como una indicación del origen empresarial y que, en consecuencia, una imitación de dicho clip podría constituir una infracción al derecho de marcas. Tribunal Regional Superior de Frankfurt (Alemania), Marken R 2001, 162-Product catalogue. 
se trate, o en "formas" u otros elementos que den una ventaja funcional o técnica al producto o al servicio al cual se aplican. El TJCA ha venido aplicando -y explicando- las referidas causales en relación a signos tridimensionales, desde hace ya varios años ${ }^{67}$.

Como se explicó anteriormente, en la interpretación prejudicial objeto de análisis, se intercambia -siguiendo el método interpretativo de la ratio legis- el concepto "textura" por el de "forma", distinguiendo, como causales de irregistrabilidad, entre las texturas usuales y las impuestas por la naturaleza o función del producto. Si bien, desde hace bastante tiempo, el TJCA ha venido desarrollando la presente causal de irregistrabilidad a propósito del registro de signos tridimensionales $^{68}$ y figurativos ${ }^{69}$, esta es la primera oportunidad en que aplica el principio de no funcionalidad a signos no visibles. Las texturas funcionales son superficies genéricas o cuya utilización resulta imprescindible para que un determinado producto posea la naturaleza y las cualidades que le son propias y sirva para la obtención de los resultados que se pretenden lograr o permita la satisfacción de las necesidades que con el mismo se persiguen ${ }^{70}$.

A este respecto, cabe recordar que el tipo de formas o texturas necesarias en relación con los productos, sus envases, envolturas o envoltorios, son aquellas que indefectiblemente deberán usarse en el mercado. Esta necesidad está dada porque la propia naturaleza o la función del respectivo producto o servicio no permiten que tenga otra forma o textura ${ }^{71}$. El principio de no funcionalidad impide la protección por el derecho de marcas de signos consistentes en formas o texturas que resulten esenciales para el uso y el propósito del producto o servicio correspondiente, es decir, de formas o texturas necesarias para la obtención de un resultado técnico ${ }^{72}$.

El TJCA explica los dos supuestos contemplados en la última parte del literal (c) del artículo 135 de la Decisión 486. De conformidad con la normativa comunitaria andina, el TJCA había venido clasificando, a propósito de las marcas tridimensionales ${ }^{73}$ y figurativas ${ }^{74}$, las formas necesarias en: (i) "formas" - o texturas- impuestas por la naturaleza, y (ii) "formas" -o texturas-impuestas por la función del producto. El TJCA afirma que las texturas impuestas por la naturaleza son aquellas

$67 \mathrm{Al}$ respecto, cabe destacar la interpretación prejudicial expedida dentro del Proceso 163-IP-2013 de 2 de octubre de 2013 , en la cual el TJCA abordó, en sus consideraciones (C) el tema de la irregistrabilidad de signos por consistir exclusivamente en "formas" usuales, o en "formas" impuestas por la naturaleza o la función del producto. Véase además, las decisiones recaídas en los Procesos 24-IP-2013 de 20 de marzo de 2013 y 86-IP-2014 de 19 de noviembre de 2014.

68 Véase, por ejemplo, las interpretaciones prejudiciales expedidas en los Procesos 24-IP-2013 de 20 de marzo de 2013, 163IP-2013 de 2 de octubre de 2013 y 86-IP-2014 de 19 de noviembre de 2014.

69 Véase, acerca de la aplicación del principio de no funcionalidad a signos figurativos, las decisiones recaídas en los Procesos 7-IP-2012 de 4 de julio de 2012 y 122-IP-2013 de 16 de julio de 2013.

70 En ese sentido, Carlos Fernández-Novoa, Fundamentos de Derecho de marcas (Madrid: Editorial Montecorvo), 85; citado, por ejemplo, en la interpretación prejudicial emitida en el Proceso 61-IP-2006 de 28 de junio de 2006, 8.

71 Véase, por ejemplo, la interpretación prejudicial pronunciada en el Proceso 163-IP-2013 de 2 de octubre de 2013, 12.

72 Véase en ese sentido, el documento preparado por la Secretaría del Comité Permanente sobre el Derecho de Marcas, Diseños Industriales e Indicaciones Geográficas de la OMPI, Nuevos tipos de marcas, 2-3.

73 Véase, por ejemplo, la interpretación prejudicial recaída en el Proceso 195-IP-2007, publicado en la Gaceta Oficial 1611 del 21 de abril de 2008; que cita a la interpretación prejudicial pronunciada en el Proceso 61-IP-2006, publicado en la Gaceta Oficial 1387 del 23 de agosto de 2006.

74 Véase, por ejemplo, las interpretaciones prejudiciales recaídas en el Proceso 120-IP-2013, de 16 de julio de 2013, y en el Proceso 122-IP-2013, de 16 de julio de 2013. 
La interpretación prejudicial recaída en el Proceso 242-IP-2015 del Tribunal de Justicia de la Comunidad Andina Juan Manuel Indacochea Jauregui

que se desprenden de los elementos esenciales del producto. Estas serían aquellas que obligatoriamente, por su configuración y su esencia ${ }^{75}$, están ligadas a los productos, sus envases o sus envoltorios. En cambio, las texturas impuestas por la función del producto serían aquellas determinadas por la finalidad del producto que se pretende distinguir mediante el signo solicitado a registro.

El objetivo de la última causal de registro comentada es impedir el otorgamiento de un derecho exclusivo sobre tales formas o texturas, bajo el entendimiento que ello obstaculizaría la posibilidad de que otros competidores puedan ofrecer un producto que incorporase la misma función o, por lo menos, que pudiesen elegir libremente la solución técnica a adoptar para lograr el mismo fin y, por consiguiente, limitaría las posibles soluciones técnicas para que el producto logre su propio propósito. Se pretende evitar así que la protección conferida por el derecho de marcas se extienda injustificadamente, al punto de convertirse en un obstáculo para la libre oferta de productos que incorporen tales soluciones técnicas o características funcionales, perjudicando de esta manera la libre competencia. Por tanto, será irregistrable toda textura que comporte una ventaja o valor funcional, por cuanto conceder derechos exclusivos sobre tal textura crearía una barrera de entrada al mercado relevante para competidores potenciales.

En virtud de las razones anteriormente expuestas, así como de lo establecido en el literal (d) del Artículo 135 de la Decisión 486, los "elementos que den una ventaja funcional o técnica al producto o al servicio al cual se aplican” no meritarán protección alguna por parte del derecho de marcas. Por consiguiente, en caso sea registrada una marca que contenga tales elementos (e.g., colores $^{76}$, formas o texturas funcionales), éstos no deberán ser considerados a efectos del cotejo o comparación entre signos distintivos, por cuanto la distintividad intrínseca del signo habría sido reconocida por la oficina nacional competente en virtud a la apreciación del signo en su conjunto; vale decir, debido a que habría sido gracias a los elementos distintivos -no funcionales o técnicos- del signo que éste pudo ser considerado distintivo en su conjunto. De lo contrario, como se explicó precedentemente, podrían erigirse -o permitir el surgimiento de- barreras u obstáculos para la oferta de productos que incorporasen aquellas soluciones técnicas o elementos funcionales, perjudicando evidentemente la libre competencia.

El principio explicado en el presente acápite ha sido reiterado en diversas interpretaciones prejudiciales posteriores, relativas a la irregistrabilidad por falta de distintividad intrínseca de signos solicitados a registro de marcas, entre las cuales cabe mencionar las recaídas en los Procesos 128-IP-2017 y 234-IP-2017 ${ }^{77}$, en las cuales se afirmó textualmente lo siguiente:

"La razón por la que ciertos nuevos tipos de marcas resultan difíciles de proteger es porque generalmente incorporan características que cumplen una función

75 Véase, por ejemplo, el caso del aroma del perfume "Chanel No 5", cuya inscripción fue denegada en 2004 debido a tratarse de la esencia misma del producto.

76 Véase, en relación con el principio de no funcionalidad aplicado a las marcas de colores, la interpretación prejudicial recaída en el Proceso 232-IP-2015 de 2 de marzo de 2016, 28 y ss.

77 Interpretaciones prejudiciales de 24 de octubre de 2017 recaídas en los Procesos 128-IP-2017 y 234-IP-2017.

Número de página no utilizable para citas 
técnica en lugar de servir para indicar la procedencia empresarial de un producto. Los aspectos funcionales no son susceptibles de ser protegidos mediante el registro de marcas".

En relación con las marcas táctiles, un ejemplo de irregistrabilidad por funcionalidad podría comportarlo la textura particular de agarre ("grip") de guantes de nieve para distinguir justamente "guantes de esquí o de nieve" comprendidos en la clase 25 de la "Clasificación Internacional de Productos y Servicios para el Registro de Marcas” instituida por el Arreglo de Niza. De igual manera, la textura de caucho para identificar neumáticos o la superficie de agarre de una pelota de baloncesto para amparar precisamente balones deportivos, serían percibidas por el público consumidor como una característica funcional, impidiendo de esta manera identificar el origen empresarial del correspondiente producto -o servicio.

En consecuencia, la protección conferida por el registro no podrá recaer sobre la textura de un producto si se demuestra que sus elementos o características esenciales son atribuibles exclusivamente a un resultado funcional o técnico, debiendo descartarse el criterio de la "multiplicidad de formas", por encontrarse en desuso ${ }^{78}, \mathrm{y}$, fundamentalmente, por no constituir una excepción a las mencionadas causales de irregistrabilidad expresamente contemplada por la normativa comunitaria andina. Según este criterio, actualmente descartado en diversos sistemas de propiedad industrial, de existir otras texturas que permitan obtener el mismo resultado funcional o técnico, sería posible monopolizar o tener la exclusividad del signo táctil solicitado a través de derechos de propiedad industrial, en tanto otros operadores del mercado podrían seguir utilizando las demás texturas disponibles. En otras palabras, el hecho de que existan otras texturas que conlleven al mismo resultado técnico no es considerado en la actualidad como un argumento válido para el otorgamiento de un registro de marca sobre tal textura.

\section{Conclusión}

El TJCA ha venido desarrollando una vasta jurisprudencia acerca del concepto -autónomo del ordenamiento jurídico comunitario- de "juez nacional”, a propósito de la legitimidad activa de diversas autoridades encargadas de aplicar el derecho comunitario para solicitar interpretaciones prejudiciales ante el TJCA, concepto que incluso comprende autoridades administrativas como la SIC, el SENAPI o el INDECOPI ${ }^{79}$.

78 Véase, por ejemplo, la sentencia del TJCE de 18 de junio de 2002, Asunto C-299/99, Philips c/ Remington, sobre máquinas de afeitar consistentes en tres cabezas rotativas que suponían a su vez características funcionales esenciales atribuibles a un resultado técnico, por lo que su forma no pudo acceder al registro, a pesar de existir otras formas que podrían haber logrado el mismo resultado técnico.

79 A este respecto, es importante advertir que el TJCA aún no habría tenido oportunidad de pronunciarse respecto de la legitimidad activa del IEPI de la República del Ecuador para solicitar interpretaciones prejudiciales facultativas. Cf. Hugo 
La interpretación prejudicial recaída en el Proceso 242-IP-2015 del Tribunal de Justicia de la Comunidad Andina Juan Manuel Indacochea Jauregui

En aras de salvaguardar la aplicación uniforme de la normativa comunitaria, por parte de todas las autoridades que deban aplicar o interpretar la norma comunitaria en el territorio de los países miembros de la CAN, el TJCA ha venido manifestando que el concepto de "juez nacional" no debe circunscribirse tan solo a los actos que emanan de los “jueces" en sentido estricto, sino que debe ser comprensivo de actos de otras autoridades que aplican el derecho comunitario en los hechos. Por lo tanto, el concepto de "juez nacional" alcanza igualmente a toda autoridad administrativa que deba aplicar una norma comunitaria andina.

En la interpretación prejudicial recaída en el Proceso 121-IP-2014, el TJCA importa y adapta cinco criterios, establecidos por la jurisprudencia europea, que son reiterados en el pronunciamiento emitido en el Proceso 242-IP-2015, con objeto de verificar la legitimidad activa de la autoridad solicitante de la interpretación prejudicial: (i) origen legal y permanencia del órgano consultante; (ii) carácter obligatorio de su jurisdicción; (iii) carácter contradictorio del procedimiento; (iv) aplicación por parte del órgano consultante de normas jurídicas comunitarias; (v) independencia.

En relación con el cumplimiento del requisito formal de representación gráfica, el TJCA, al pronunciarse en el Proceso 242-IP-2015, consideró que, en primer lugar, esta debe darse mediante una descripción simple y, en segundo lugar, a través de la presentación, ante la oficina nacional competente, de una muestra física del objeto que contenga la textura reivindicada. Lo anterio, no excluye otras posibles formas de descripción del signo, como por ejemplo, el método braille o impresión en relieve.

De esta manera, el TJCA no solo toma en cuenta otras alternativas y nuevas técnicas de representación, sino que va más allá de solo admitir reproducciones de un signo distintas a las gráficas cuando estas sean más aptas para identificarlo, como establece la última reforma normativa europea en materia de derecho de marcas, sino que impone la obligación de presentar una muestra o espécimen físico del producto que contenga la textura solicitada a registro.

Por otra parte, el TJCA realiza una interpretación extensiva de la noción de "forma", referida en los literales (c) y (d) del artículo 135 de la Decisión 486, incluyendo en la misma a todo aspecto externo del producto -y no únicamente su envase o continente- susceptible de ser captado por los consumidores, incluso la "textura" del producto, así como de su envase o cualquier otro continente del producto.

El TJCA advierte que las oficinas nacionales competentes y los jueces nacionales deberán tener especial cuidado al momento de realizar el examen de fondo de las solicitudes de registro de nuevos tipos de signos, manifestando que es preciso realizar un examen minucioso, verificando que ninguno de los elementos o características reivindicadas sean parte de la naturaleza del producto que se pretende identificar (literal (c) del artículo 135), ni que sean usuales o de uso

Gómez Apac, "The Principle of Primacy of Reality in Judicial Precedent on Industrial Property of the Court of Justice of the Andean Community", The Trademark Reporter (INTA), 108: 5.

Número de página no utilizable para citas 
común en la clase internacional a la cual pertenece el producto amparado (literal (c) del artículo 135), ni que cumpla una finalidad o función técnica; es decir, que no se reivindiquen elementos que respondan a la propia función del producto (literal (c) del artículo 135) o aquellos que otorguen una ventaja funcional o técnica al producto (literal (d) del artículo 135).

Cabe destacar que la interpretación prejudicial recaída en el Proceso 242-IP-2015 constituye la primera oportunidad en que el TJCA desarrolla debidamente - de manera suficiente- el principio de no funcionalidad, propio del derecho de marcas, esencial a fin de examinar la registrabilidad de nuevos tipos de marcas o signos. Además, es también la primera ocasión en que un tribunal o corte internacional se pronuncia acerca de la registrabilidad de una marca táctil o de textura.

Finalmente, es importante recordar la necesidad de complementar lo establecido en la Decisión 486, en el sentido de desarrollar a través de la legislación nacional de los países miembros, principalmente, el principio de no funcionalidad -además de la prohibición de registro de formas o texturas de uso común- como causal de irregistrabilidad de marcas por falta de distintividad intrínseca, aplicada no solo a signos tridimensionales, figurativos o de colores, sino también a signos no visibles, de conformidad con la jurisprudencia previamente comentada y los criterios jurisprudenciales recogidos en la interpretación prejudicial recaída en el Proceso 242-IP-2015. 